\title{
Design Guidelines for Interactive Multimedia Learning Environments to Promote Social Inclusion
}

Dr. David J. Brown, Dr. Heather M. Powell, Steven Battersby, James Lewis, Nick Shopland and Masha Yazdanparast.

The Greenhat Interactive Research Team, Department of Computing, Nottingham Trent University, Newton Building, Burton Street, Nottingham, NG1 4BU. +(44) 1159486019 .

Emails: david.brown@ntu.ac.uk or heather.powell@ntu.ac.uk 


\section{Structured Abstract}

Purpose: There is a continuing need for guidelines to aid in the design of Interactive Multimedia Learning Environments (IMLE) to promote effective learning. The project introduced in this paper looks at an important subset of this problem, the design of interactive learning environments to promote social inclusion.

Method: A consortium of six partners contributed toward defining learning material to develop a range of work based skills, including horticulture, IT and catering. These were then developed into IMLE prototypes. Formative evaluation of these prototypes then revealed a range of usability problems, which were grouped into generic types and frequency of occurrence.

Results: The most important and frequently occurring problems were used to distil a set of design guidelines for the development of effective IMLE. The results from this Usability Content Analysis were also used to refine the initial prototypes to improve their usability and effectiveness.

Conclusions: These guidelines called the Greenhat Design Guidelines can be adopted for use by all multimedia developers aiming to promote the social inclusion of vulnerable or socially disadvantaged groups of people. The refined IMLE can be accessed via the Greenhat Server to improve the employment-related skills of socially excluded people.

\section{Introduction}

The aim of the Flexible Learning Systems project is to develop a range of IMLE to be used by socially excluded people for the development of real employment opportunities. 
This project is funded under the European ADAPT Programme, which aims to up-skill those at risk of redundancy using Information and Communication Technologies (ICT). This is one of several European initiatives aimed at producing rehabilitation and associated technologies, in response to a strong North American market created through Disability Act Legislation [1].

The use of ICT in supporting people, who for a variety of reasons face social exclusion, in an industrial setting is not a new idea [2]. Areas of employment skill development tackled within this project include horticulture, catering, IT and self-determinism. These skills have been identified as being valuable to people whose jobs are threatened, where they can be used to sustain employment.

The Flexible Learning Systems package has been implemented by the Greenhat Research Team, within the Department of Computing, at The Nottingham Trent University. This is a collaborative project, with national partners within the U.K. including Mencap, Thrive, Wigan and Leigh College, Kaleidoscope and the Welsh Centre for Learning Disabilities.

This consortium represented the needs of a wide range of people who for various reasons face social exclusion. Our collective definition of social exclusion is that the risk of nonparticipation is high if:

a) They are young and their parents are poor or unemployed

b) They are members of certain minority ethnic groups 
c) They are in particular circumstances that create barriers to participation:

- They have a learning difficulty

- They have a physical disability

- $\quad$ They are carers

- they are teenage parents

- $\quad$ They are homeless

- They are or have been in care

- $\quad$ They have a mental illness. [3]

This is a wide definition and places considerable challenge on any design team attempting to cater for the range of needs within such a grouping of people. It appears that poverty plays a major part in social exclusion. Percy-Smith [4] points out, however, that poverty is not the only problem. Duffy, Walker and Walker [5] agrees, stating that social exclusion also encompasses an individual's inability to participate effectively in economic, social, political and cultural life, and, in some characterisations, alienation and distance from mainstream society.

Social exclusion is a problem that can start early in an individual's life. According to BBC1's television programme 'Eyes of a child update', up to 600 children are excluded permanently or temporarily every school day in Britain [6]. The vast majority are either tutored at home, placed in referral units, or eventually return to mainstream education. In response the UK government has set up the social exclusion unit that is established and run from the Cabinet office [7]. The primary focus of the unit is on those who are 
disadvantaged, have low aspirations, are at risk of dropping out, failing to achieve, or not making a successful transition to further education, training or employment.

In order to develop IMLE to develop employment based skills in socially excluded people a methodology which embraced user centred design principles should be embraced. It was decided that a methodology, previously developed by the Greenhat Research team at the Nottingham Trent University, could be adapted for use. This methodology had been successfully employed to guide the development and evaluation of the Virtual City, a series of virtual learning environments to teach independent living skills to people with learning disabilities. It was judged to be too specific to the needs of people with a learning disability [8], although was a useful starting point, especially the components advocating strong user centred design. The adapted methodology design was also heavily influenced by guidelines such as the World Wide Web Accessibility Initiative [9] and Universal Design [10].

\section{Method:}

Some of the partners involved in the project had been previously involved in joint projects to develop paper based learning materials and packages to promote employment based skills. These packages were seen as being a useful starting point in developing interactive multimedia based learning materials to help sustain employment. Their use 
therefore affected the design of the existing development methodology [4], culminating in a project methodology presented in figure 1.

[Insert figure 1 about here]

\subsection{Usability Content Analysis}

In line with our development methodology, a process of formative evaluation was undertaken with twelve of the IMLE prototypes. These included food hygiene prototypes for catering skills, introduction to computer hardware and basic Microsoft prototypes for IT skills, amenity horticulture prototypes and key skills prototypes. This formative evaluation process was interleaved throughout the project life cycle, with the results being used to inform the ongoing development process. Usability content analysis questionnaires were devised, to pinpoint usability difficulties in the prototypes at an early development stage. These questionnaires concentrated on issues such as navigability, aesthetics and the language level of prototypes.

The formative evaluation meetings were held at three monthly intervals throughout the project life cycle. These were in reality expert review sessions, with testers drawn from the flexible learning systems consortium. The experimental methodology for the usability content analysis of each prototype was:

- Form a testing sub-group of six members from the testing meeting members, with no more than two members from each partner in the consortium.

- Assign a member of the testing group to record points on the usability questionnaires. 
- Set five tasks as group activity within each prototype.

- Record usability problems.

- Feedback these usability problems to the whole testing meeting.

- Isolate commonly occurring usability problems experienced by all testing groups across prototypes.

- Distil design guidelines from the most commonly occurring usability problems.

\section{Results:}

\subsection{The Greenhat Educational Server}

The Flexible Learning Systems tutors are available online at www.flexiblelearningsystems.net, via the Greenhat Educational Server at NTU. The philosophy of making these materials freeware is that as with most Computer Aided Learning (CAL) packages aimed at the rehabilitation of socially excluded people, the prime aim is to promote their maximum accessibility. Effective delivery is a crucial phase in producing educational multimedia systems, and failure to do so is the single greatest cause of system failure [11]. Free online delivery is an ideal delivery mechanism, as an alternative to expensively distributed CD-ROM based systems.

Some examples of screenshots from some of the 30 IMLE (in total) produced as part of the Flexible Learning systems projects are shown in figure 2.

[Insert figure 2 about here] 
An example of usability content analysis is now summarised, illustrating how the Basic Food Hygiene Tutor was evaluated (one of the twelve packages fully evaluated in this way). Two testing sub-groups were formed, each given a range of tasks to carry out within the Basic Food Hygiene Tutor. Usability problems were recorded on questionnaires by a nominated recorder within each group. Subsequent analysis of these questionnaires, triangulated with other verbal feedback, allowed the collation of a range of commonly occurring usability problems to be grouped generically. When this process was carried out for twelve of the IMLE prototypes (IT, Horticulture, Catering and Key Skills tutors), it became possible to spot the frequently re-occurring usability problems, and from these distil a set of design guidelines. These guidelines will optimise the design of IMLE to promote the employment prospects of socially excluded people.

\subsection{Formation of testing sub-groups:}

The testing sub-groups were selected in line with the procedure specified in the methods section. These are shown in table 1 for the usability content analysis of the Basic Food Hygiene Tutor.

[Insert table 1 about here]

\subsection{Food hygiene usability tasks}

A range of tasks were set for each of these testing sub-groups, within the basic food hygiene 
multimedia tutor. These included washing hands and wearing appropriate clothing before entering a food preparation area, food preparation and clearing the workstation after preparation.

\subsection{Recorded usability problems}

Table 2 illustrates the usability problems that the two-testing sub-groups (A and B) experienced in using the prototype food hygiene tutor. Some comments have been moved from their original sections (where placed by the nominated recording person) to other, more appropriate sections - indicated by appending its former section number. Items in bold indicate that they were addressed in revision of the prototype, items in italics are comments that required no action, all other items have been taken into account for future work, but could not be addressed within the original development plan time scale.

[Insert table 2 about here] 


\subsection{Feedback on commonly occurring usability problems and suggested prototype}

revisions

The following points summarise the usability problems and suggested changes to the Basic Food Hygiene prototype based on results from the usability content analysis.

\subsubsection{Graphics}

- Replace the background graphic for non-copyright material. Varied backgrounds would be better. A lot going on one page, background and images 'merge'.

- Larger, clearer icons and buttons are required. Place controls centrally.

- Hazard symbols are good, but could be they larger?

- More 3D, animated and actual images are required. Pictures and videos would be better than line drawings.

- Graphical instead of numerical total recording user performance are required at the end of user interactions.

The overall recommendations were to replace the background with something less likely to "clutter" the screen, and introduce additional backgrounds to reflect the intention to make all IMLE modular. Modify, enlarge and centralise the control buttons, add sounds for mouse-overs. Replace a proportion of the line drawn graphics with photographic or other images.

\subsubsection{Text}

- Highlight keywords. 
- More movement is required. Text needs to be interesting and provide an impact but not be busy.

- Adopt a linear, textual approach. Text may work better if shorter and more structured sections. It should be 'chunked' per topic, in a modular approach. Could be more flexible and allow more user direction. A lot of material covered, feels lengthy. Section (a7) is large, without much information.

- "How food is poisoned" is too busy, present steps one by one.

The overall recommendations were to highlight keywords and pace screen text with narration and animation of the text. Break up some of the lengthy and busy areas. Attempt to modularise the prototype along the lines of:

1. Food Handlers

2. Food Poisoning Symptoms

3. How Food is Poisoned (Poisonous Plants - Chemicals - Germs)

4. Review

5. Interactive Test.

\subsubsection{Sounds}

- The "Well done" sound effect is overdone.

- Place reminders and voice cues on buttons.

- Use different voices for different purposes. Use different voices to break the monotony of sound tracks, and use voices of both genders.

- Voiceover script needs to match the text.

- Have an option to turn sound on and off. Don’t make comprehension reliant on the use of sound alone. 
The overall recommendations for this prototype were to review use of sound and to introduce one or two other speakers, with additional sound effects. The IMLE should also allow the user to disable sounds.

\subsubsection{Interactions}

- The game playing and ‘move on’ interaction options are not clear.

- The 'type in name' interaction option is unclear.

The overall recommendation was to rationalise the movement through the prototype and correct inappropriate interactions.

\subsubsection{Additions}

- A user guide is required at the start of the prototype (to introduce navigation and interactions options).

- Help and contact numbers need to be listed.

- A minimum hardware specification needs to be defined and clearly announced. Advice should be given if the system is not up to specification.

- $\quad$ Other I/O methods need to be offered, such as a touch screen, joystick, and keyboard interaction option.

- Feedback should be also available to the user in a hardcopy as well as electronic format, as evidence of achievement.

The overall recommendations included devising a guide to introduce users to the key navigation and interaction paradigms, the addition of help contact information and identify the minimum specification for running the prototype, plus some troubleshooting tips. Other recommendations included developing a range of 
alternative joystick and keyboard interaction possibilities, as well as developing some printable support material.

\section{Design Guidelines}

As each of the IMLE prototypes under went usability content analysis (12 packages), leading to their refinement, a set of the most frequently occurring usability problems encountered by the testing groups emerged. From this process a set of design guidelines were distilled, to guide the development of interactive multimedia tutors to promote the social inclusion of individuals by developing the employment skills.

The formation of these design guidelines was heavily influenced by ethos and presentation of the World Wide Web Accessibility Guidelines to promote the accessibility of the web by people with sensory, cognitive and physical impairments. Each of the guidelines, termed the Greenhat Design Guidelines, is presented with design recommendations for programmers and designers of interactive learning packages in a series of priority levels. The lower the priority number, the more imperative the need to follow the guideline to ensure accessibility. This set of design guidelines also states the design considerations needed to achieve the key objectives of promoting the development of employment based skills. The guidelines are divided into sections. Each section contains an abstract and a list of key points in order of priority, followed by any notes. 


\subsection{Priority Levels}

Each design point is given a level of priority based on its potential impact on accessibility.

Within Priority 1 a software developer or designer of interactive learning material must satisfy this design point. This is a basic requirement to achieve accessibility, otherwise a range of socially excluded people will find it impossible to access information.

Within Priority 2 a software developer or designer of interactive learning material should satisfy this design point. This is required to achieve wide accessibility and enjoyment of the product. Otherwise a range of socially excluded people will find it difficult to access information and find the product of little educational use.

Within Priority 3 a software developer or designer of interactive learning material may address this design point. If these points are implemented then the product will successfully fulfil the need for full accessibility and an enjoyable learning tool for socially excluded people.

The main sections of the Greenhat design guidelines are now introduced derived from a process of usability content analysis on a range of IMLE aimed at promoting employment and key skills. It should be noted that this is the first draft of these design guidelines, and subsequent evaluation of other IMLE aimed at the development of employment and other skills in socially excluded people, will provide richer and wider feedback with which to strengthen them. Other IMLE currently in development 
include those to develop numeracy, literacy and IT skills in socially excluded people which will be accessed via a range of cyber cafes within disadvantaged areas.

Usability content analysis of these additional IMLE will allow us to further refine the guidelines and widen their scope of application.

\subsection{Accessibility Issues}

This section defines the design issues concerned with creating an accessible product for a wide range of disabilities. Some issues in this section may be applicable to other sections.

Priority 1:

- $\quad$ Provide simple user interaction mechanisms for navigation. i.e., single-click mouse.

- $\quad$ Provide a speech alternative of all buttons and key text within the IMLE.

Priority 2:

- $\quad$ Provide an icon, text and speech for all buttons.

- $\quad$ Provide speech representation of text and images in all IMLE.

- $\quad$ Try to set a limit of up to two sentences of text for each page of the IMLE.

- $\quad$ More complex user input should only be included in an 'Advanced Topics' section and then only kept to a minimum.

Priority 3:

- $\quad$ Provide the user with a choice of input devices - mouse, joystick, keyboard.

- $\quad$ Provide an option to display sign language.

- $\quad$ Provide an option to turn any sound on or off. 


\subsection{Navigation}

Navigation should be clear and consistent so that the user feels comfortable with the

IMLE. It must also be flexible so users of different abilities can progress at different speeds throughout the IMLE. The navigational elements can vary to match the aesthetics of their module as long as they conform to some basic rules.

Priority 1:

Each IMLE requires:

- A menu that allows the user to jump to any module in any order.

- An exit button to exit the application.

Each module within the IMLE requires:

- Contents that allow the user to jump to any topic in any order.

- An exit button to return to the IMLE Menu.

Each page within the module requires (except games and VR):

- A next button to progress to next page.

- A back button to progress to previous page.

- An exit button to return to the module Contents.

Where the software can automatically progress through the pages of the IMLE:

- This must be at a speed suitable for people with literacy problems.

- $\quad$ Provide explicit prompts when the IMLE requires user input to progress.

Priority 2:

- Disable navigation buttons during compulsory learning objectives.

- Provide an optional user-guide on how to navigate through the package.

- Provide an index that allows the user to jump to any topic.

- Provide an option to turn on or off the automatic page progression. 
Priority 3:

- Provide hot-keys for fast navigation between topics and pages for teachers and tutors using the IMLE with a user.

\subsection{Pedagogic Structure}

The user may be accompanied by a teacher or tutor, or alternatively they may use the IMLE alone, especially if it is used online. The product needs to perform pedagogic tasks such as reinforcement and reward so that the user is aware that they are making progress.

Priority 1:

- Reward - compliment them when they have completed a task.

- $\quad$ Reinforcement - recap areas covered.

- Test - ensure the user has learnt the lessons.

Priority 2:

- Module reinforcement - recap subjects learnt.

- Progress Indication - change the look of the Contents to reflect topics covered.

- $\quad$ Topic reinforcement - recap key points.

- Reward regularly. 
- User ability options - allow selection/number of topics dependant on users.

- Progress hard copy - allow printout of results / rewards / "certificates".

\section{Priority 3:}

- Calculation of user ability - use feedback to access ability and change the progress.

Notes: 


\section{Buttons}

Abstract:

Buttons are an integral part of the navigational element of the product. As they are the primary interface with the user it is crucial they conform to some basic rules. See the section on accessibility on other design guidelines on buttons.

Priority 1:

- Clear meaning.

- Unambiguous.

- Consistent throughout package:

- Screen position.

- Consistent throughout module:

- Shape.

- Size.

- Colours.

- An indication when the cursor is over a button.

- Large buttons.

Priority 2:

- Minimum size of 80 pixels radius or square.

- Exit Button in top - right corner.

- Next Button in bottom - left corner. 
- Back Button in bottom - right corner.

- Grey-out button when disabled.

Priority 3:

- Match aesthetics of module.

- Should have an icon with text underneath or textual hint.

- The cursor should change to a pointing hand when over a button.

Notes: 
Hotspots

\section{Abstract}

A hotspot is any selectable area that the user can click in to navigate or perform an action.

Priority 1:

- An indication when the cursor is over the selectable area.

Priority 2:

- A speech representation of all hotspots.

- A text representation near the hotspot or a textual hint on mouse over.

- Hotspots should be of a reasonable size, larger than the image if necessary.

Notes: 
Aesthetics

\section{Abstract}

Aesthetics effect the quality of the product. Some elements must be consistent so that the user feels comfortable with the product while other elements must vary so that the product is exciting and enjoyable to learn from.

Priority 1:

- Maximum of three sentences per page. If necessary split text over 2 pages.

Priority 2:

- Background should be consistent within topic.

- Backgrounds should have similar "look" throughout module.

- Vary voices of speech.

- Vary media types between vector graphics, photo, video and 3D.

Priority 3:

- Highlight keywords in text.

- Record speech from people with abilities similar to the users.

Notes: 


\section{Games}

\section{Abstract}

Games are an exciting way to learn. For users with learning disabilities it is essential that the game is easy to play and rewarding. Similarly it is important that games do not require too much dexterity otherwise it will exclude users with physical disabilities.

Priority 2:

- Fun and exciting to play for all users.

- Regular rewards.

Notes:

Click games are easier than dragging games. 


\section{Screen Properties}

\section{Abstract}

This is the overall screen properties.

Priority 1:

- Minimum requirement of 800x600 resolution.

Priority 2:

- Software should be centred on higher resolutions.

- Black surround on higher resolutions if full screen.

Priority 3:

- Full screen.

Notes:

\section{Conclusions:}

\section{Acknowledgements:}

7. References:

[1] (Carruthers et al, 1993). 
[2] (Barkvik et al, 1998).

[3] Ogden C. Teaching numeracy to the socially excluded. Undergraduate thesis, Department of Computing, The Nottingham Trent University 2001.

[4] Percy-Smith J. Policy responses to social exclusion. Buckingham, Open University Press 2000.

[5] Walker A and Walker C. Britain divided. London: CPAG ltd 1997.

[6] Eyes of a child update, 2001. TV, BBC1, $7^{\text {th }}$ March 2001.

[7] DFEE web site.

[8] Brown DJ. Presence, 2001

[9] World Wide Web Accessibility Initiative 2001.

[10] Waters C. Universal Web Design. New Riders Publishing, Indianapolis, Indiana, USA, 1997.

[11] Boyle T. Design for multimedia learning. 1997; p218, 


\section{Multimedia Package Specification}

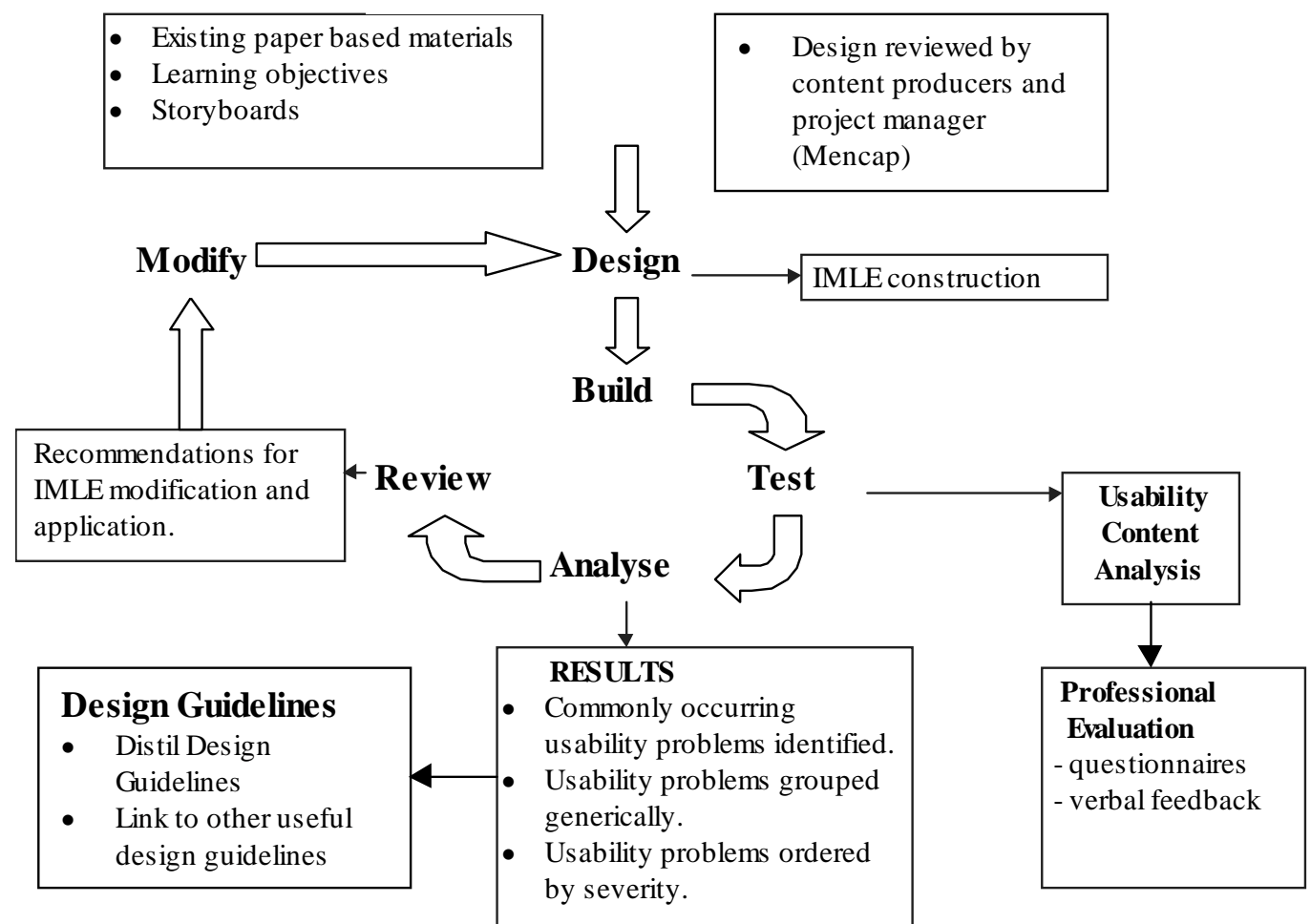

Figure 1: The development methodology for IMLE to promote the development of employment related skills in socially excluded people. 

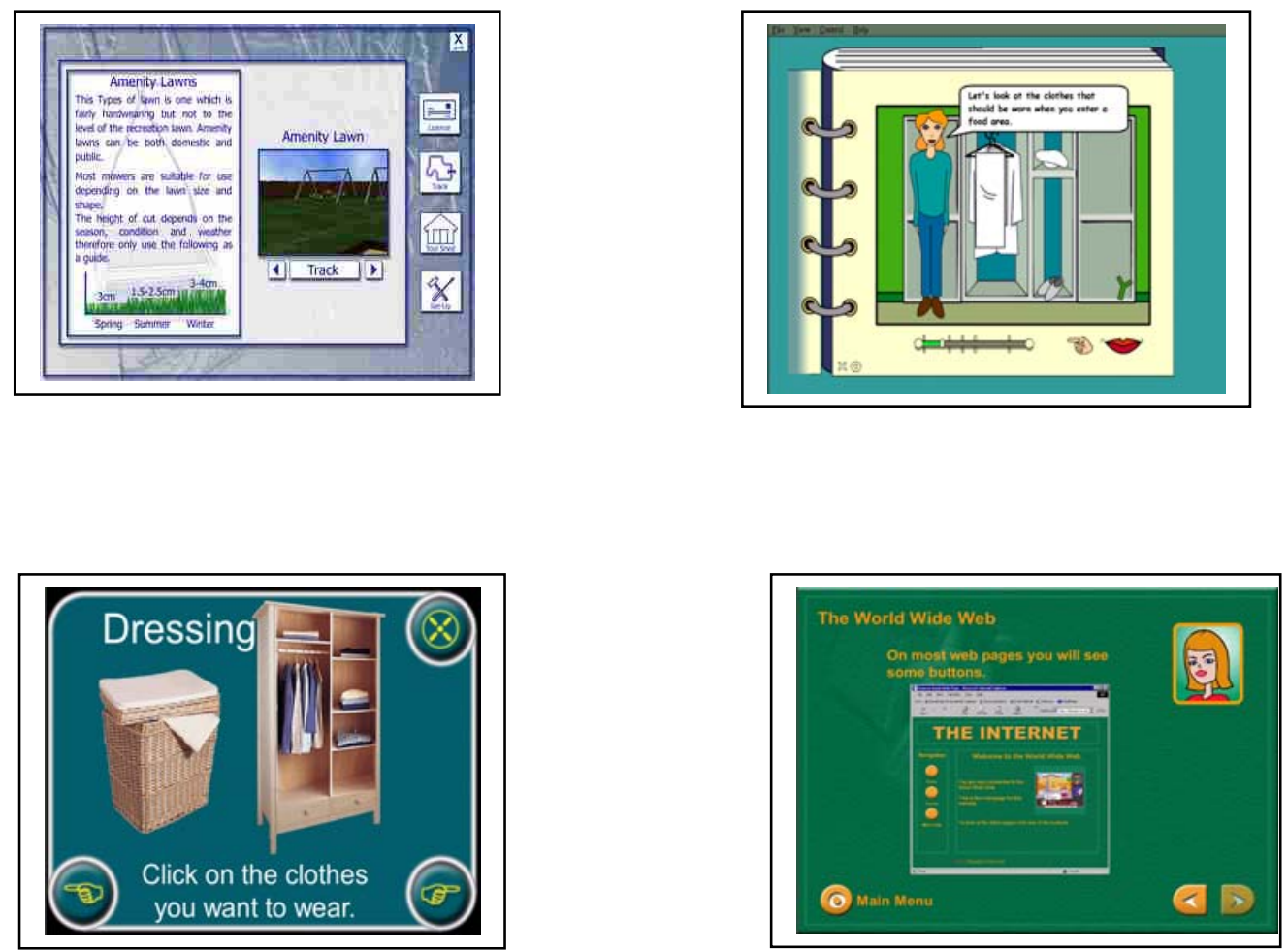

Figure 2: Some screenshots from the IMLE to promote the employment skills of socially excluded people. 


\begin{tabular}{ll}
\hline Group A & Group B \\
\hline Joe Guthrie (M) & Jacqui Lewis (M) \\
Simon Veasey (K) & Tim Clamp (K) \\
Mahsa Yazdanparast (NTU) & Steven Battersby (NTU) \\
Christine Massey (W\&L) & Angeline Sudworth (W\&L) \\
Neil Kelly (TH) & Chris Martin (TH) \\
Heather Powell (NTU) & Nick Shopland (NTU)
\end{tabular}

\begin{tabular}{|ll|}
\hline Key: & M=Mencap \\
& K=Kaleidoscope \\
& NTU=Nottingham Trent University \\
W\&L=Wigan and Leigh College \\
TH=Thrive
\end{tabular}

Table 1: The testing sub-groups for the usability content analysis of the Basic Food Hygiene Tutor. 
1. Navigation

Larger, clearer icons/buttons

( $A$ \& B)

Place controls centrally

(B)

User guide at the start (introduce navigation \& interactions)

Need user guide at start; include guide to interactive events

(B) - 5

Help/contact numbers

(A)

Play game \& move on options not clear

(A)

Type in name interaction unclear

Reminders/voice cues on buttons

User initialisation; choose how screen is to look (e.g. text/Widget)

Embedded tutor/character (assistant)

(B)

2. I/O Methods

Other methods: touch screen, joystick and keyboard

Voice activation

(B)

3. Aesthetics

Varied backgrounds would be better

Highlight keywords

(A)

Hazard symbols good - could be larger?

Pictures \& videos instead of line drawings

More 3D/animated/actual images

Graphical instead of numerical total at end of interaction

A lot going on on one page - background/images 'merge'

Needs to be interesting with impact but not busy, e.g. Germs text movement (B)

More movement

Use different voices to break monotony

(A) -4

Corporate - common buttons/presentation

More fun elements

(A) 
4. Linguistics

Voiceover script needs to match text

"How food is poisoned" - too busy - present one by one

(A) - 3

Use different voices for different purposes

(A)

"Well done" overdone - sounds for pass/fail

(A) - 3

Too many "Well done"s

(B) - 5

Package is text heavy

Simplistic in parts - Milk from a cow; show contamination at different stages

(A) -8

\section{Pedagogic Structures}

Feedback - Hardcopy?

Hard copy element for feedback

(B) - 2

Paper evidence

(A) - 9

Paper content - main points

(A) -9

Text copy needs to be made computer friendly

Feedback - How to reward \& how often

Strategy for review where there is misunderstanding - alternative approaches

Feedback - interactivity/reinforcement - to what extent?

Applause good (as reinforcement)

\section{Package Size}

A lot of material covered; feels lengthy

Large section (a7) without much information

(A) - 7

Not sure until video etc in

(A)

7. Interaction level

Could be more flexible/modular

Could be more flexible $\&$ allow more user direction

(B) - 8 
9. Flexibility

Turn sound on/off - not reliant on sound

10. System Requirements

Minimum spec needs to be defined and clearly announced

Advice if system not up to spec.

11. Additional Considerations

Replace background graphic for non-copyright material

Table 2: Recorded usability problems for the Basic Food Hygiene Tutor. 